EASD

Procedia
EURODYN 2020

XI International Conference on Structural Dynamics M. Papadrakakis, M. Fragiadakis, C. Papadimitriou (eds.) Athens, Greece, 23-26 November 2020

\title{
COUPLED ITM-FEM APPROACH FOR THE ASSESSMENT OF THE MITIGATION EFFICIENCY OF FINITE AND INFINITE OPEN TRENCHES AND INFILLED BARRIERS
}

\author{
Julian Freisinger ${ }^{1}$, Gerhard Müller ${ }^{1}$ \\ ${ }^{1}$ Chair of Structural Mechanics, Technical University of Munich \\ Arcisstr. 21, 80333 Munich, Germany \\ e-mail: julian.freisinger@tum.de, gerhard.mueller@tum.de
}

Keywords: Soil Dynamics, Soil-Structure-Interaction(SSI), Integral Transform Method, Finite Element Method, Wavenumber-Frequency Domain, Vibration Mitigation

\begin{abstract}
In order to determine the wave propagation in unbounded media considering the interaction of structures with the soil and thus also their influence on the resulting wave field, an appropriate model is necessary. A efficient approach to account for the infinite extension of the soil by the analytical solutions of the Integral Transform Method (ITM) and for the irregular geometries of the structures by the Finite Element Method (FEM) is presented. The coupled ITM-FEM approach is applied for the assessment of the mitigation efficiency of infinite open or infilled trenches using a computationally profitable $2.5 \mathrm{D}$ approach. For spatially limited trenches a complete three dimensional model is used. Amplitude reduction factor and insertion loss are calculated for different combinations of soil and barrier material. The accuracy of the method is shown by comparison to analytical solutions and literature results.
\end{abstract}

ISSN:2311-9020 @ 2020 The Authors. Published by EASD Procedia.

Peer-review under responsibility of the Organizing Committee of EURODYN 2020.

doi: $10.47964 / 1120.9232 .19194$ 


\section{INTRODUCTION}

In the modern urban environment, vibrations are caused by a variety of sources such as rail and road traffic, industrial- or construction machinery. These vibrations are transmitted into the soil causing disturbances in adjacent structures and annoyance of nearby residents. In order to attenuate the disrupting effects, mitigation measures can be installed at the source or the receiving structure. However, as a big part of the vibratory energy is transported by Rayleigh waves propagating in an area close to the ground surface, introducing suitable wave barriers in the transmission path leads to an appropriate reduction in the amplitude of the elastic waves on the side of the barrier averted from the source. Usually both, open and soft or stiff infilled trenches act as vibration isolating screens by reflecting and diffracting the incident waves.

A comprehensive amount of studies on the mitigation efficiency of open and infilled trenches can be found in literature. First field tests on the screening effect of rectangular open and bentonite filled trenches were conducted by Dolling [1]. Further measurements were performed by Woods [2], who clearly distinguished between active isolation, installing the open trench in the near field very close to the source, and passive isolation placing the trench in the far field, where the Rayleigh wave dominates the wave field. Numerical studies investigating the amplitude reduction of Rayleigh waves by open or concrete filled trenches using the Finite Element Method (FEM) in the frequency domain were presented by Segol et al. [3] and Haupt [4]. Later, the Boundary Element Method (BEM) was employed by Emad and Manolis [5] as well as Beskos et al. [6], Dasgupta et al. [7], Leung et al. [8], who used it to investigate the efficiency of open and infilled trenches due to harmonic sources. Ahmad and Al-Hussaini [9] performed an extensive parametric study on the effects of the dimensions and the filling material of trenches and barriers situated in a homogeneous as well as a layered soil. To be able to include more complex barrier and source compositions, still satisfying the radiation condition for infinite media, different FEM-BEM formulations were developed and used to predict the reduction of ground vibrations by wave barriers due to moving loads [10,11,12]. Mitigation measures aiming on the reduction of railway induced vibrations can be assumed to be longitudinally invariant. Coulier et al. [13] and François et al. [14] used a coupled 2.5D FEM-BEM approached based on [15] to investigate the effect of soft and stiff wave barriers, open trenches and subgrade stiffening next to the track.

In this paper an efficient domain decomposition method to model the three dimensional SoilStructure-Interaction (SSI) is presented using a coupled Integral Transform Method (ITM) Finite Element Method approach. The infinite soil is described by the analytical solutions of the ITM avoiding any non-physical reflections at artificial boundaries, whereas the FEM is used to model structures of more complex geometry and material distribution. Fully or partially embedded cylindrical or spherical cavities can be modeled, thus enabling the coupling of finite or longitudinally invariant structures within a matching FEM subsystem.

The outline of the paper is as follows. In section 2 firstly the fundamental solutions are introduced and superposed in order to obtain the stiffness matrix of the ITM subsystems in the respective reference system. Subsequently the dynamic stiffness of the FEM subsystems is presented, followed by the coupling of the substructures. A validation of the proposed method is shown in section 3 by comparison with the analytical solution of the homogeneous halfspace. In section 4, a 2.5D ITM-FEM approach is used to model infinite open trenches and infilled barriers. First, the results for a concrete filled trench and subgrade stiffening next to the track are checked against literature results. Second a comparison of the mitigation efficiency of very 
soft or stiff barriers and an open trench is presented. Furthermore a 3D ITM-FEM model is used to investigate the screening efficiency of a spatially limited open trench for different load positions and frequencies. Finally the results for the length invariant open trench are directly opposed to those for the finite open trench.

\section{ITM-FEM FORMULATION FOR ELASTODYNAMICS}

\subsection{Fundamental solutions}

The dynamic behavior of an linear elastic, homogeneous and isotropic continuum can be described by the Lamé differential equation, which consists of three coupled partial differential equations

$$
\left.\mu u^{i}\right|_{j} ^{j}+\left.(\lambda+\mu) u^{j}\right|_{j} ^{i}-\rho \ddot{u}^{i}=0
$$

with the displacement field $u^{i}$, the Lamé constants $\mu$ and $\lambda$ and the density $\rho$. Using a Helmholtz decomposition $u^{i}=\left.\Phi\right|^{i}+\left.\Psi_{l}\right|_{k} \varepsilon^{i k l}$ and thereby expressing the displacement field by the sum of the gradient of a scalar field $\Phi$ and the rotation of a vector field $\Psi$, the system of equations turns into three decoupled wave equations in dependency of the wave velocities of the compressional $c_{p}$ and the shear wave $c_{s}$ (setting $\Psi_{z}=0 \mathrm{cp}$. [16]).

$$
\left.\Phi\right|_{j} ^{j}-\frac{1}{c_{p}^{2}} \ddot{\Phi}=\left.0 \quad \Psi_{i}\right|_{j} ^{j}-\frac{1}{c_{s}^{2}} \ddot{\Psi}=0
$$

The fundamental solutions for the systems halfspace, fullspace with cylindrical cavity and fullspace with spherical cavity are derived from (2) transforming the equations into the appropriate basis and then applying the respective local and non-local boundary conditions.

The system halfspace is solved in Cartesian coordinates $(x, y, z)$ by using a threefold Fourier transform into the wavenumber-frequency domain $\left(k_{x}, k_{y}, z, \omega\right)$. Thus the coupled partial differential equations can be transformed into three decoupled ordinary differential equations that can be solved analytically with an exponential approach [17]:

$$
\begin{aligned}
& \hat{\Phi}=A_{1} e^{\lambda_{1} z}+A_{2} e^{-\lambda_{1} z} \\
& \hat{\Psi}_{\alpha}=B_{\alpha 1} e^{\lambda_{2} z}+B_{\alpha 2} e^{-\lambda_{2} z}
\end{aligned}
$$

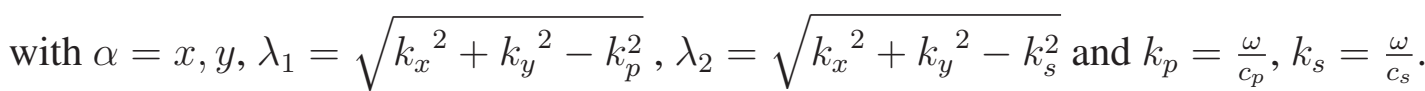

The system fullspace with cylindrical cavity is solved in cylindrical coordinates $(x, r, \varphi)$ under application of a Fourier transform with respect to $x \circ \bullet k_{x}$ and $t \circ \bullet \bullet \omega$ as well as performing a Fourier Series expansion along the circumferential direction $\varphi \rightarrow n$. Furthermore the vector field $\Psi=\Psi \mathrm{g}^{1}+\left.\chi\right|^{j} \varepsilon_{i j 1} \mathbf{g}^{i}$ is expressed by two independent scalar fields. The respective system of equations is solved with Hankel functions of first and second kind [18]:

$$
\begin{aligned}
& \hat{\Phi}\left(k_{x}, r, n, \omega\right)=C_{1 n} H_{n}^{(1)}\left(k_{1} r\right)+C_{4 n} H_{n}^{(2)}\left(k_{1} r\right) \\
& \hat{\psi}\left(k_{x}, r, n, \omega\right)=C_{2 n} H_{n}^{(1)}\left(k_{2} r\right)+C_{5 n} H_{n}^{(2)}\left(k_{2} r\right) \\
& \hat{\chi}\left(k_{x}, r, n, \omega\right)=C_{3 n} H_{n}^{(1)}\left(k_{2} r\right)+C_{6 n} H_{n}^{(2)}\left(k_{2} r\right)
\end{aligned}
$$

with $k_{1}^{2}=k_{p}^{2}-k_{x}^{2}$ respectively ${k_{2}}^{2}={k_{s}}^{2}-k_{x}{ }^{2}$.

The system fullspace with spherical cavity is solved in spherical coordinates $(r, \varphi, \vartheta)$. All quantities on the spherical boundary are transformed into series of spherical harmonics $(\varphi, \vartheta \rightarrow$ 
$m, l)$ and a Fourier transform into the frequency domain is carried out. Thus the wave equations are solved in the $(r, m, l, \omega)$ domain by spherical Hankel functions of first and second kind [19]:

$$
\begin{aligned}
& \hat{\Phi}(r, m, l, \omega)=C_{1 l m} h_{m}^{(1)}\left(\left|k_{p}\right| r\right)+C_{4 l m} h_{m}^{(2)}\left(\left|k_{p}\right| r\right) \\
& \hat{\psi}(r, m, l, \omega)=C_{2 l m} h_{m}^{(1)}\left(\left|k_{s}\right| r\right)+C_{5 l m} h_{m}^{(2)}\left(\left|k_{s}\right| r\right) \\
& \hat{\chi}(r, m, l, \omega)=C_{3 l m} h_{m}^{(1)}\left(\left|k_{s}\right| r\right)+C_{6 l m} h_{m}^{(2)}\left(\left|k_{s}\right| r\right)
\end{aligned}
$$

For all three systems local stress boundary conditions are applied respectively on the halfspace surface $\Lambda$, the cylindrical cavity surface $\Gamma_{c}$ or the spherical cavity surface $\Gamma_{s}$. Additionally employing the Sommerfeld radiation condition [20] the vector of unknown coefficients $\mathbf{C}_{i}$ can be determined for each system separately and the stresses and displacements due to unit load states at the surfaces can be calculated by $\hat{\boldsymbol{\sigma}}_{i}=\hat{\mathbf{K}}_{i} \mathbf{C}_{i}$ and $\hat{\mathbf{u}}_{i}=\hat{\mathbf{H}}_{i} \mathbf{C}_{i}$, where $i=h s, c, s$ stands for the different fundamental systems and $\hat{\mathbf{K}}_{i}, \hat{\mathbf{H}}_{i}$ are the matrices including the fundamental solutions, kinematic and material relations linking the unknowns with the stresses and the displacements. A detailed derivation of the solutions of the fundamental systems as well as the matrices for the calculation of the $\hat{\boldsymbol{\sigma}}_{i}$ and $\hat{\mathbf{u}}_{i}$ can be found in [21, 22] and [23].

\subsection{Superposition of fundamental systems}

The superposition of two of the fundamental systems each is now used to satisfy the boundary conditions of the complete system at both surfaces. Therefore the halfspace is superposed with the fullspace with cylindrical cavity resulting in a halfspace with cylindrical cavity. Furthermore the halfspace is superposed with the fullspace with spherical cavity to obtain the system of a halfspace with spherical cavity. For this reason fictitious surfaces $\delta \Lambda$ resp. $\delta \Gamma_{j}$ (with $j=c, s$ for the cylinder resp. the sphere) are introduced into the fundamental systems allowing a superposition at the common surfaces as depicted in Figure 1. Thus, loading the halfspace with unit stress states $\hat{\boldsymbol{\sigma}}_{\Lambda}$ at $\Lambda$ for each wavenumber combination $\left(k_{x}, k_{y}\right)$, the stresses $\hat{\boldsymbol{\sigma}}_{\delta \Gamma_{j}}^{(\Lambda)}$ at the fictitious surface $\delta \Gamma_{j}$ can be determined. The other way round, the stresses $\hat{\boldsymbol{\sigma}}_{\delta \Lambda}^{\left(\Gamma_{j}\right)}$ at $\delta \Lambda$ due to unit stress states $\hat{\sigma}_{\Gamma_{j}}$ at $\Gamma_{j}$ for each of the respective series members ( $n$ resp. $m, l$ ) can be calculated. The sum of the stresses at $\Lambda$ and $\Gamma_{j}$ has then to be equal to the external loads $\hat{\boldsymbol{p}}_{\Lambda}$ and $\hat{p}_{\Gamma_{j}}$ acting on the complete system. Thus the scaling factors $\mathbf{C}_{\mathbf{I T M}}$ for the unit stresses and the
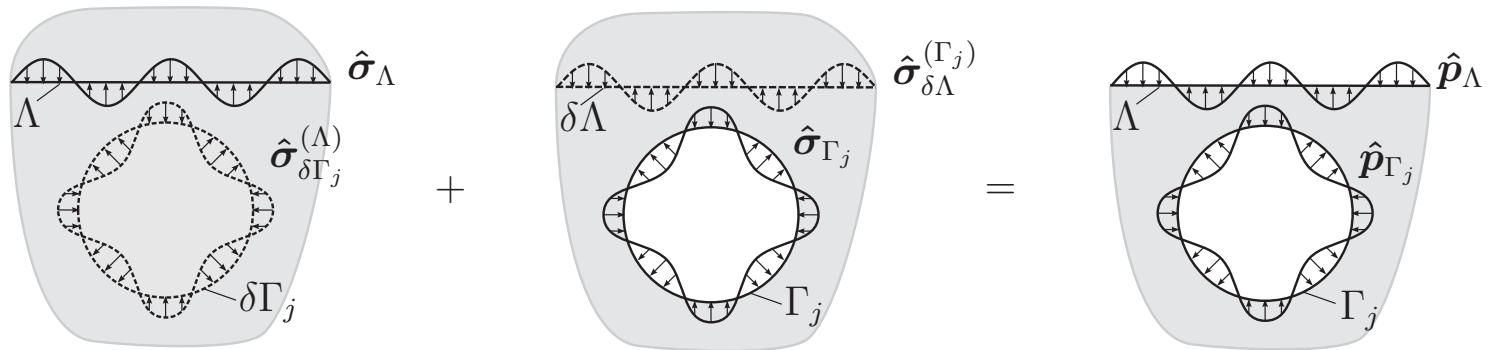

Figure 1: Fictitious surfaces and stresses at the surfaces due to unit stress states.

stresses at the fictitious boundaries, gathered in $\hat{\mathbf{S}}_{\text {ITM }}$, can be determined in dependency of the total external load $\hat{\mathbf{P}}_{\mathbf{I T M}}$ on $\Lambda$ and $\Gamma_{j}$ as:

$$
\mathrm{C}_{\text {ITM }}=\hat{\mathbf{S}}_{\text {ITM }}^{-1} \hat{\mathbf{P}}_{\text {ITM }}
$$


Subsequently, also the resulting displacements $\hat{\mathbf{u}}_{\mathrm{ITM}}$ at $\Lambda$ and $\Gamma_{j}$ can be calculated from the displacements due the unit stresses, gathered in $\hat{\mathbf{V}}_{\text {ITM }}$, scaled with the amplitudes $\mathrm{C}_{\text {ITM}}$ :

$$
\hat{\mathbf{u}}_{\text {ITM }}=\hat{\mathbf{V}}_{\text {ITM }} C_{\text {ITM }}
$$

Combining equations (11) and (12) the stiffness matrix $\hat{\mathbf{K}}_{\text {ITM }}=\hat{\mathbf{S}}_{\text {ITM }} \hat{\mathbf{V}}_{\text {ITM }}^{-1}$ of the ITM subsystem for a halfspace with cylindrical or spherical cavity is obtained giving a direct relation between the displacements and the external loads:

$$
\underbrace{\left[\begin{array}{cc}
\hat{\mathbf{K}}_{\Lambda \Lambda_{\mathrm{ITM}}} & \hat{\mathbf{K}}_{\Lambda \Gamma_{\mathrm{j} \text { ITM }}} \\
\hat{\mathbf{K}}_{\Gamma_{\mathrm{j}} \Lambda_{\mathrm{ITM}}} & \hat{\mathbf{K}}_{\Gamma_{\mathrm{j}} \Gamma_{\mathrm{j} \text { ITM }}}
\end{array}\right]}_{\hat{\mathbf{K}}_{\mathrm{ITM}}} \underbrace{\left(\begin{array}{c}
\hat{\mathbf{u}}_{\Lambda_{\mathrm{ITM}}} \\
\hat{\mathbf{u}}_{\Gamma_{\mathrm{j}} \text { ITM }}
\end{array}\right)}_{\hat{\mathbf{u}}_{\mathrm{ITM}}}=\underbrace{\left(\begin{array}{c}
\hat{\mathbf{P}}_{\Lambda_{\mathrm{ITM}}} \\
\hat{\mathbf{P}}_{\Gamma_{\mathrm{j} \text { ITM }}}
\end{array}\right)}_{\hat{\mathrm{P}}_{\mathrm{ITM}}}
$$

The systems halfspace with cylindrical or spherical indentation can be derived analogously by shifting the cavity to the halfspace surface. Further details can be found in [24].

\subsection{Finite Element Implementation}

The FEM structures that shall be coupled to the previously introduced ITM subsystems need to match with the shape of the respective cavity surface $\Gamma_{j}$. Therefore the dynamic stiffness matrix of a FEM mesh with a cylindrical and a spherical outer boundary have to be computed. Applying the 2.5D approach, the stiffness of the halfspace with cylindrical cavity was calculated in the $\left(k_{x}, \omega\right)$ domain. This allows to represent the 3D response on a two dimensional mesh. Thus quadrilateral finite elements with linear shape functions are used to discretize the cross section of the FEM substructure. However, the FEM formulation has to be adopted to the wavenumber frequency domain as presented in [18]. To enable a direct stiffness coupling with the ITM later on, the nodes need to be equally distributed along the cylindrical boundary.

For the spherical FEM structure usual three dimensional, eight node solid elements can be used and the dynamic stiffness matrix is computed in Cartesian coordinates and in the frequency domain as well $(x, y, z, \omega)$. The FEM nodes on the spherical boundary must be situated on the intersections of latitudes and longitudes to be able to transform the FEM stiffness matrix into the basis of the ITM subsystem for the coupling. Thereby the $z$-coordinates of the latitudes are chosen as the Gauß points along the diameter and the longitudes are equally distributed along the circumference [22].

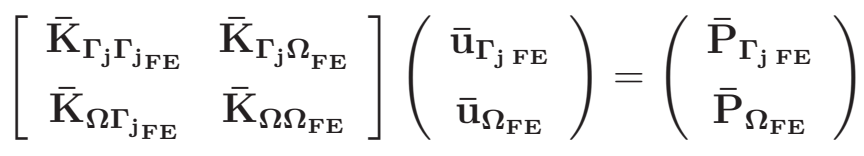

\subsection{Coupling of Substructures}

The coupling of the ITM and the FEM substructure is carried out at the cylindrical respectively the spherical boundary of the substructures. On the coupling surface the continuity of displacements as well as the equilibrium of forces are used as transition conditions. As reference system for both cases the basis of the ITM subsystem is chosen. Therefore the dynamic stiffness matrix of the cylindrical FEM subsystem on $\Gamma_{c}$ needs to be transformed into the $\left(k_{x}, r, n, \omega\right)$ domain by applying a twofold Fourier transform as well as a Fourier series expansion along the circumference. The spherical FEM structure is transformed into the $(r, m, l, \omega)$ domain by transforming the dynamic stiffness on $\Gamma_{s}$ into spherical coordinates, conducting a Fourier transform into the frequency domain and developing it into series of spherical harmonics. The FEM 
formulation inside the structure $\Omega$ is kept in the $(x, y, z, \omega)$ domain.

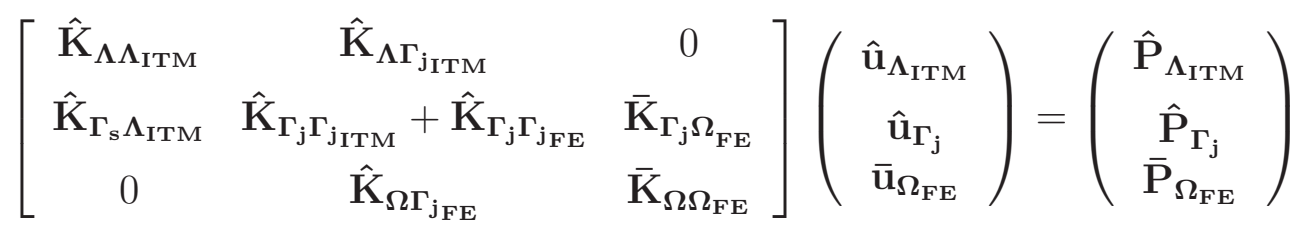

\section{VALIDATION}

The systems halfspace with partly embedded cylindrical or spherical cavity are verified in this section by a comparison with the analytical solution of a homogeneous halfspace. Therefore in the coupled ITM-FEM system the cavity is homogeneously filled with finite elements. Same material parameters are chosen for the FEM substructure and the surrounding soil modeled by the ITM. The system is excited by a vertical harmonic load with $f=12 \mathrm{~Hz}$ at the halfspace surface $\hat{\mathbf{P}}_{\boldsymbol{\Lambda}}$ with block size $2 \times 2 \mathrm{~m}$ and amplitude $P_{0}=1 \frac{\mathrm{N}}{\mathrm{m}^{2}}$ at $y_{\text {load }}=-10 \mathrm{~m}$ outside of the FEM inclusion with radius $R=4 \mathrm{~m}$. (Figure 2). The soil material parameters are chosen as Soil A given in Table 2.

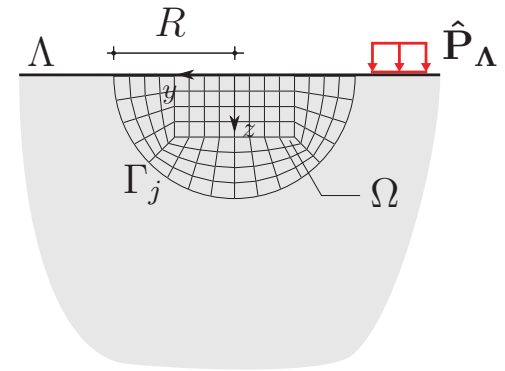

(a)

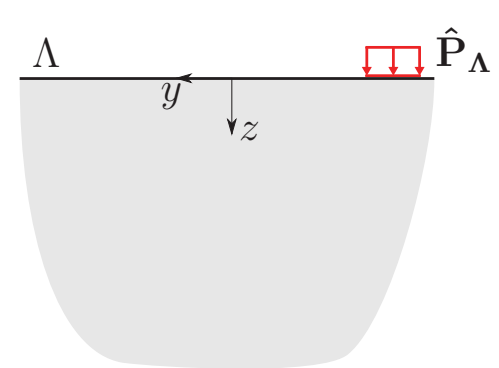

(b)

Figure 2: Setup for comparison of coupled ITM-FEM system (a) with analytical solution for homogeneous halfspace (b).

The results for the absolute value of the vertical displacement on the halfspace surface are presented normalized with the load resultant in Figure 3. A very good agreement of the coupled ITM-FEM approach with the benchmark solution can be stated for both, the $2.5 \mathrm{D}$ and the $3 \mathrm{D}$ model.

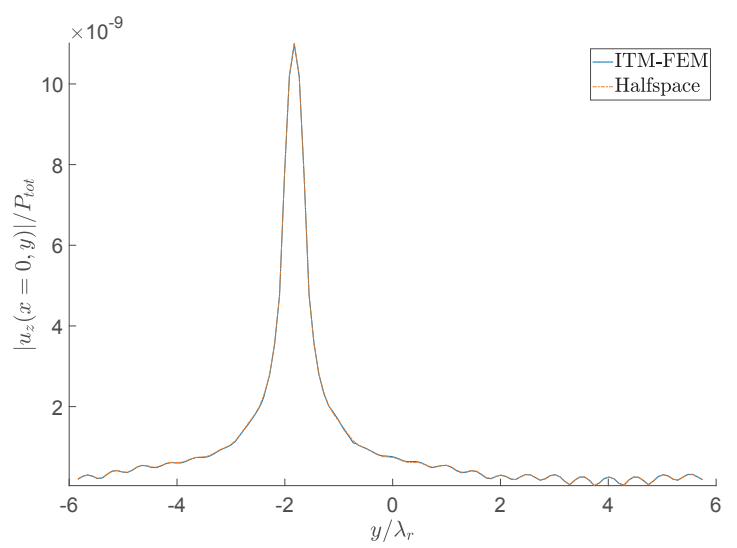

(a)

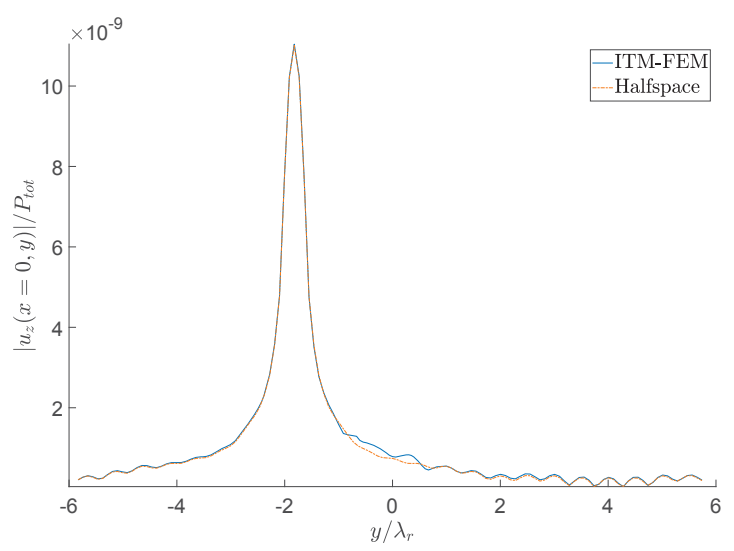

(b)

Figure 3: Comparison of $\left|u_{z}(x=0, y, z=0, \omega)\right|$ between analytical solution for homogeneous halfspace and (a) halfspace with cylindrical FEM inclusion (2.5D), (b) halfspace with spherical FEM inclusion (3D). 


\section{NUMERICAL RESULTS}

\subsection{Infinite open trenches and infilled barriers (2.5D)}

In this section numerical results for length invariant open trenches and infilled barriers are presented applying the proposed 2.5D ITM-FEM approach for a halfspace with cylindrically shaped FE substructure. Firstly the results gained with the proposed methodology for a concrete filled trench are compared with those available in published literature. Second the effect of subgrade stiffening as wave impeding barrier is shown followed by a comparison of the mitigation efficiency and the mechanism of action of open trenches and soft or stiff barriers.

\section{Concrete filled trench}

In this example the Rayleigh wave diffraction by a rectangular trench, infilled with concrete, in the transmission path, as depicted in Figure 4a, is investigated with the ITM-FEM approach. The results are compared with those obtained by Haupt [4] with the FEM, and Beskos et al. [6] with a constant element based BEM implementation. As the literature results were calculated for a $2 \mathrm{D}$ case, the ITM-FEM solution was also done only for wavenumber $k_{x}=0$, which means constant conditions in longitudinal direction $x$. A vertical harmonic load with amplitude $P_{0}=1 \frac{\mathrm{N}}{\mathrm{m}^{2}}$ and a width of $b_{\mathrm{y}, \text { load }}=2 \mathrm{~m}$ is applied at a distance of $y_{\text {load }}=-16 \mathrm{~m}$ from the trench with a frequency of $f=30 \mathrm{~Hz}$ resulting in a Rayleigh wavelength of $\lambda_{r}=3.18 \mathrm{~m}$. The width and depth of the barrier were chosen to $w_{\mathrm{tr}}=1.25 \mathrm{~m}$ and $d_{\mathrm{tr}}=3.25 \mathrm{~m}$, prescribed by the FE discretization. Therefore the normalized dimensions result to $W_{\mathrm{tr}}=0.39, D_{\mathrm{tr}}=1.02$ and $L_{\text {load }}=5.03$, which almost coincide with the dimensions used in [4, 6]. The further discretization and geometry parameters are given in Table 1 as well as the material parameters of the soil (Soil B) and the concrete filling (Infill A) in Table 2.

\begin{tabular}{l|c|c|c|c|c|c}
\hline & $N_{x}=N_{y}$ & $B_{x}=B_{y}$ & $N_{\varphi}$ & $R$ & $d_{x}=d_{y}=d_{x, \mathrm{FE}}$ & $d_{y, \mathrm{FE}}$ \\
\hline Concrete filled trench & $2^{9}$ & 128 & 128 & 8 & 0.25 & 0.125 \\
Subgrade stiffening & $2^{9}$ & 128 & 96 & 8 & 0.25 & 0.333 \\
Comp. soft-stiff-open & $2^{8}$ & 64 & 192 & 8 & 0.25 & 0.166 \\
\hline
\end{tabular}

Table 1: Discretization and geometry parameter for coupled ITM-FEM approach.

\begin{tabular}{l|c|c|c|c|c|c|c}
\hline & $E\left[\frac{\mathrm{N}}{\mathrm{m}^{2}}\right]$ & $\nu[-]$ & $\rho\left[\frac{\mathrm{kg}}{\mathrm{m}^{3}}\right]$ & $\zeta[-]$ & $c_{p}\left[\frac{\mathrm{m}}{\mathrm{s}}\right]$ & $c_{s}\left[\frac{\mathrm{m}}{\mathrm{s}}\right]$ & $c_{r}\left[\frac{\mathrm{m}}{\mathrm{s}}\right]$ \\
\hline Soil A & $26.00 \cdot 10^{6}$ & 0.30 & 2000 & 0.05 & 132.5 & 70.8 & 65.7 \\
Soil B & $46.12 \cdot 10^{6}$ & 0.25 & 1720 & 0.03 & 179.5 & 103.6 & 95.3 \\
Soil C & $21.60 \cdot 10^{7}$ & 0.33 & 2000 & 0.05 & 400.5 & 201.7 & 188.0 \\
Infill A & $15.81 \cdot 10^{8}$ & 0.25 & 2356 & 0.05 & 914.6 & 528.1 & 485.8 \\
Infill B & $15.12 \cdot 10^{8}$ & 0.248 & 2000 & 0.025 & 950.7 & 550.3 & 506.1 \\
Infill C & $30.00 \cdot 10^{9}$ & 0.2 & 2600 & 0.01 & 3580.5 & 2192.6 & 1998.9 \\
Infill D & $25.00 \cdot 10^{4}$ & 0.4 & 700 & 0.05 & 27.6 & 11.3 & 10.6 \\
\hline
\end{tabular}

Table 2: Material parameters of different soils and infill materials

In Figure $4 \mathrm{~b}$ the amplitude reduction factor $A_{r}$ for the absolute value of the vertical displacement $\left|u_{z}(x=0, y, z=0, \omega)\right|$ at the halfspace surface is shown at $x=0$ over the dimensionless distance $y / \lambda_{r} . A_{r}$ is defined as the amplitude in presence of the trench $\left|u_{z, \mathrm{tr}}\right|$ divided by the amplitude in absence of the trench $\left|u_{z \text {,ref }}\right|$. Thus values of $A_{r}$ smaller than one mean a reduction of the vibration amplitudes of $\left(1.0-A_{r}\right) \cdot 100$ percent. Significant amplifications are observed 
in front of the trench caused by the constructive or destructive interference of the incident and reflected Rayleigh waves due to their phase difference [9], which is indicated by the peak distances being very close to $\lambda_{r} / 2$. On the load remote site, a substantial amplitude reduction due to the concrete barrier of $50-70 \%$ is achieved. A very good agreement between the literature results and the ITM-FEM approach can be stated.

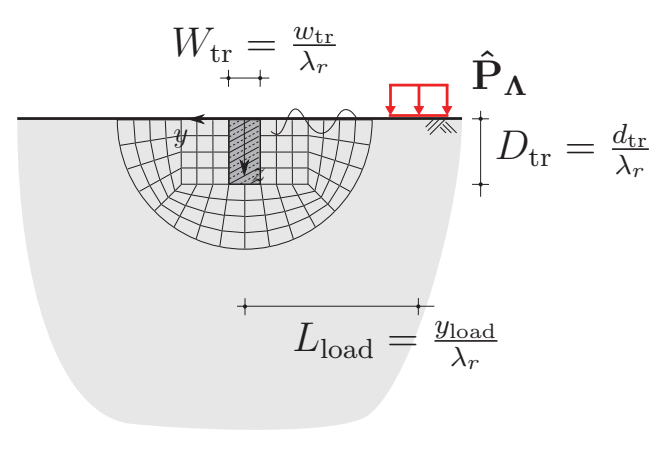

(a)

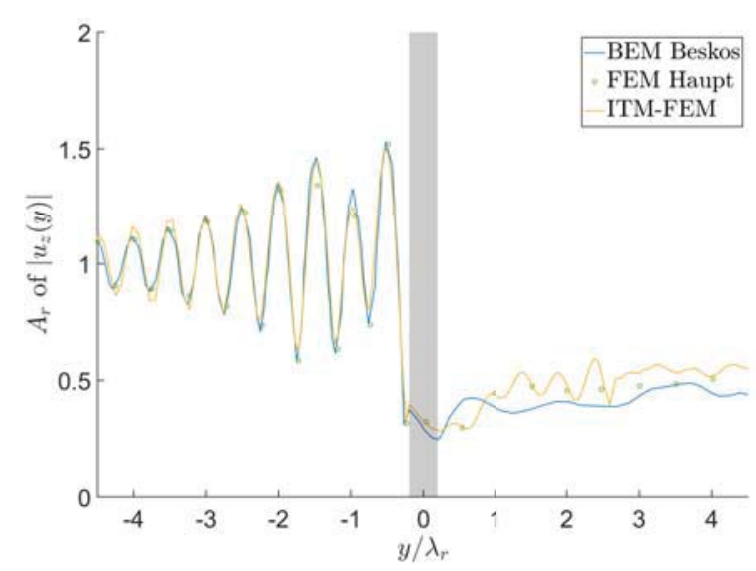

(b)

Figure 4: (a) ITM-FEM model for concrete filled trench in transmission path. (b) Comparison of ITM-FEM results for amplitude reduction factor $A_{r}$ of the vertical displacement $\left|u_{z}\right|$ with BEM solution of Beskos et al. [6] and FEM solution of Haupt [4].

\section{Subgrade stiffening as wave impeding barrier}

In this section the 2.5D ITM-FEM approach is used to investigate the efficiency of subgrade stiffening as mitigation measure in the transmission path. The setup depicted in Figure 5a is adopted from Coulier et al. [13], who used a 2.5D FEM-BEM methodology to analyze subgrade stiffening next to the track for the mitigation of railway induced vibrations. A block of stiffened soil (Infill B) with a width and depth of $w_{\mathrm{tr}}=d_{\mathrm{tr}}=2 \mathrm{~m}$ located within a homogeneous halfspace (Soil C) is investigated. The discretization parameters are given in Table 1. The material parameters for the surrounding and the stiffened soil, leading to the same wave velocities as in [13], are presented in Table 2. A quadratic, harmonic block load with $P_{0}=1 \frac{\mathrm{N}}{\mathrm{m}^{2}}$ and a width of $b_{\mathrm{x}, \text { load }}=b_{\mathrm{y} \text {,load }}=1 \mathrm{~m}$ is applied at a distance of $y_{\text {load }}=-6 \mathrm{~m}$ from the barrier. The absolute value of vertical displacements and the insertion loss $\mathrm{IL}_{z}$ over the total surface were evaluated for the reference case $\left|u_{z, \text { ref }}\right|$ without subgrade stiffening and the situation in presence of the stiffened block $\left|u_{z, t r}\right|$. Therefore positive values of the insertion loss

$$
\overline{\mathrm{L}}_{z}(x, y, z=0, \omega)=20 \log _{10} \frac{\left|u_{\mathrm{z}, \mathrm{ref}}\right|}{\left|u_{\mathrm{z}, \mathrm{tr}}\right|}[\mathrm{dB}]
$$

indicate a reduction compared to the reference case without mitigation measure.

Coulier et al. [13] showed by an investigation of the insertion loss in the wavenumberfrequency domain, that the block of stiffened soil can act as a wave impeding barrier. Considering the stiffened block as infinitely long Timoschenko beam, it was demonstrated that the wave impeding effect depends strongly on the relation of the projection of the Rayleigh wave length in the soil in longitudinal direction $\lambda_{x}$ and the free bending wave length in the beam $\lambda_{b}$. The transmission of plane waves in the soil with $\lambda_{x}<\lambda_{b}$ is impeded, as the response of the 
beam is then dominated by its bending stiffness and the amplitude decreases proportionally to $k_{y}^{-4}$ for a given frequency.

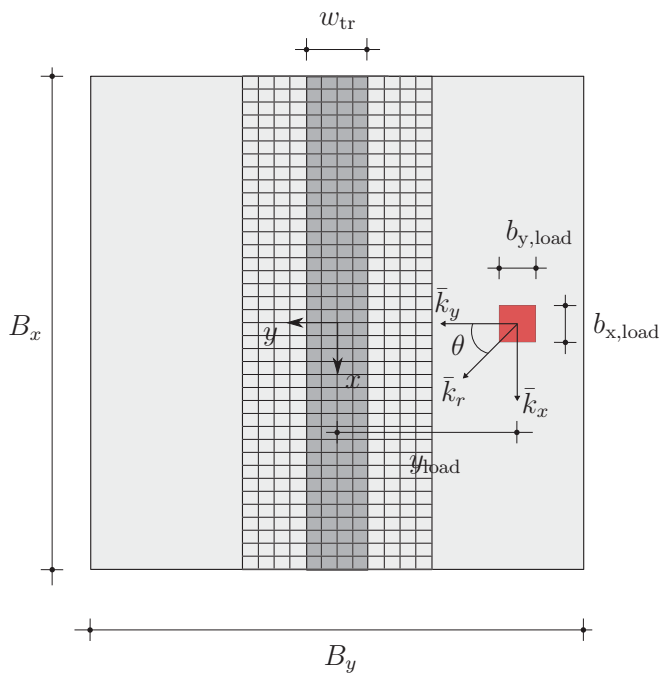

(a)

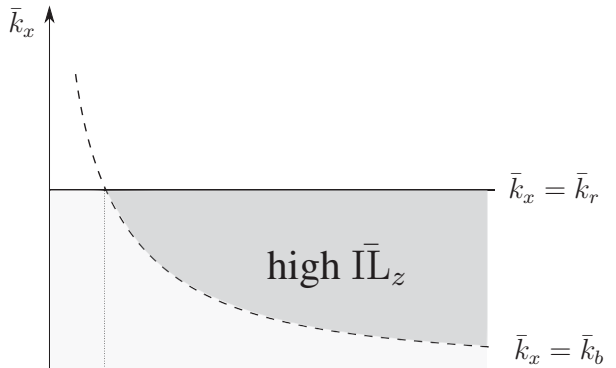

low $\overline{\mathrm{I}}_{z}$

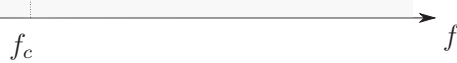

(b)

Figure 5: (a) Topview of setup for comparison of coupled ITM-FEM system with 2.5D FEM-BEM model of Coulier et al. [13]. (b) Schematic sketch of the dispersion curves of the free bending wave in a Timoschenko beam and the Rayleigh wave in a homogeneous halfspace.

Figure 5b shows a schematic sketch of the insertion loss $\overline{\mathrm{I}}_{z}\left(\bar{k}_{x}, y, z=0, \omega\right)$ over the dimensionless wavenumber $\bar{k}_{x}=k_{x} c_{s} / \omega$ and the frequency $f$ as presented in [13]. A high insertion loss is obtained for wavenumbers $\bar{k}_{x}$ smaller than the dimensionless Rayleigh wavenumber $\bar{k}_{x}=\bar{k}_{r}=c_{s} / c_{r}$ and bigger than the free bending wavenumber $\bar{k}_{b}$. The contribution of wavenumbers $\bar{k}_{x}>\bar{k}_{r}$ to the total response and especially the response in lateral direction is quite limited. This is due to the fact, that the value of the transfer function of a homogeneous halfspace in the wavenumber frequency domain for wavenumbers $k_{x}^{2}+k_{y}^{2}>k_{r}^{2}$ is quickly decreasing with increasing wavenumbers and thus, as the propagation direction in the $x-y$ plane is given by $\theta=\tan ^{-1}\left(\bar{k}_{x} / \bar{k}_{y}\right)$, for $\bar{k}_{x}>\bar{k}_{r}$ the waves traveling in $y$-direction have only comparatively small amplitudes. The propagation direction of the Rayleigh wave, which makes up the largest part of the overall response results as $\theta_{r}=\sin ^{-1}\left(\bar{k}_{x} / \bar{k}_{r}\right)$. For wavenumbers $\bar{k}_{x}<\bar{k}_{b}$ follows $\lambda_{x}>\lambda_{r}$ and thus the Rayleigh wave is able to propagate through the stiffened block with low transmission loss, whereas for $\bar{k}_{x}>\bar{k}_{b}$ the waves are impeded by the barrier because $\lambda_{x}$ gets smaller than $\lambda_{b}$. Therefore a critical frequency $f_{c}$, when the mitigation measure becomes effective, can be determined by the intersection of the Rayleigh wave and the free bending wave dispersion curves [13]:

$$
f_{c}=\frac{c_{r}^{2}}{2 \pi} \sqrt{\frac{\rho A}{E I}} \sqrt{\frac{E \mu \kappa}{\left(E-\rho c_{r}^{2}\right)\left(\mu \kappa-\rho c_{r}^{2}\right)}}
$$

where $E$ is the Young's modulus, $\mu$ the shear modulus, $\rho$ the density, $A$ the cross sectional area and $I$ the moment of inertia of the beam. $\kappa$ is the shear coefficient and can be set to $\kappa=5 / 6$ for rectangular cross sections. $c_{r}$ is the Rayleigh wave velocity in the soil. This critical frequency strongly depends on the stiffness contrast between the soil and the block of stiffened soil. Thus subgrade stiffening is more effective in soft soils. For the given material parameters the critical frequency results to $f_{c}=12.37 \mathrm{~Hz}$. 
The propagation direction of the Rayleigh waves is given by $\theta_{r}$. Beyond a critical angle of $\theta_{c}=\sin ^{-1}\left(\bar{k}_{b} / \bar{k}_{r}\right)$ and assuming $\omega>\omega_{c}$, the wavenumbers $\bar{k}_{x}$ are bigger than $\bar{k}_{b}$ and thus $\lambda_{x}<\lambda_{b}$ holds. Therefore the impinging plane waves with $\theta>\theta_{c}$ are impeded by the barrier. The critical angle in dependency of the frequency is given by [13] as:

$$
\theta_{c}(f)=\arcsin \left(c_{r} \sqrt{\rho\left(E+\mu \kappa \pm \sqrt{(E-\mu \kappa)^{2}+\frac{4 E(\mu \kappa)^{2} A}{\rho I(\omega / 2 \pi)^{2}}}\right)(2 E \mu \kappa)^{-1}}\right)
$$

Figure $6 \mathrm{a}$ and $6 \mathrm{c}$ show the real part of the vertical displacement $u_{z}(x, y, z=0, \omega)$ due to a harmonic load with $f=5 \mathrm{~Hz}<f_{c}$ for the reference case and the case when subgrade stiffening was applied. The Rayleigh wave length $\lambda_{r}$ is much larger than the depth of the barrier and thus a significant part of the Rayleigh wave is able to pass beneath it. For all waves impinging at the stiffened block, $\bar{k}_{x}>\bar{k}_{b}$ holds and therefore they are not impeded. The wave field in both cases is very similar and the corresponding insertion loss $\mathrm{IL}_{z}$ in Figure $6 \mathrm{e}$ is almost zero over the whole surface. The real part of the vertical displacements for a excitation frequency of $f=30 \mathrm{~Hz}>f_{c}$ is shown in Figure $6 \mathrm{~b}$ and $6 \mathrm{~d}$. In the reference case cylindrical wave fronts are observed, whereas, if subgrade stiffening is applied, the wave field is not cylindrical anymore due to the interaction of the barrier and the soil. All waves hitting the barrier at an angle greater than the critical angle $\theta_{c}$ are impeded, leading to a significant reduction of vibrations in the areas with $\theta>\theta_{c}$. Hence, large values of the insertion loss, partly exceeding $10 \mathrm{~dB}$, are observed there. The critical angle, which results to $\theta_{c}=43.26^{\circ}$ at $f=30 \mathrm{~Hz}$, is sketched in Figure 6f.

However, below the critical angle the amplitude reduction is rather small due to the relatively shallow barrier (only $0.32 \lambda_{r}$ at $f=30 \mathrm{~Hz}$ ) and the not so pronounced stiffness contrast between the barrier and the soil. Although lines of increased $\mathrm{IL}_{z}$ can be observed in this area due to the partial destructive interference caused by the phase shift of the waves passing below the barrier and the waves transmitted through the barrier with much higher wave velocity, which appears mainly for thick barriers of lower depth [25]. For larger depth of the barrier and an increased stiffness contrast also for angles $\theta<\theta_{c}$ a significant reduction can be achieved, as more reflections occur at the left face of the barrier and less vibrations are transmitted. Furthermore a smaller part of the Rayleigh wave passes below the barrier. On account of this in the following section the mitigation efficiency for an open trench, a soft and a stiff barrier are compared, assuming adequate dimensions and a sufficient stiffness contrast.

\section{Stiff and soft barriers vs. open trench}

The vibration screening efficiency of open trenches massively depends on the trench depth. A satisfactory screening efficiency of $A_{r}<0.25$ can be achieved for depths $d_{\text {tr }}$ greater than about $1.2 \lambda_{r}$ of the soil [2]. Due to stability reasons the construction of ideal open trenches with vertical sides is limited to shallow depths. For this reason, trenches are often filled with soft material that must be able to withstand the horizontal soil stresses while still providing adequate insulation [26]. Open trenches achieve their vibration reduction effect by the reflection of the incident Rayleigh waves. As no energy can be transferred across the open trench the vibration reduction usually is higher than for infilled trenches, where a part of the wave energy is transmitted through the barrier. If a very soft infill material is used the behavior of the filled trench resembles that of open trench [27], which also yields for the mitigation efficiency.

However, the physical mechanism, which is responsible for the vibration reduction changes, when it comes to stiff barriers. Additionally to the reflected and transmitted Rayleigh waves, 


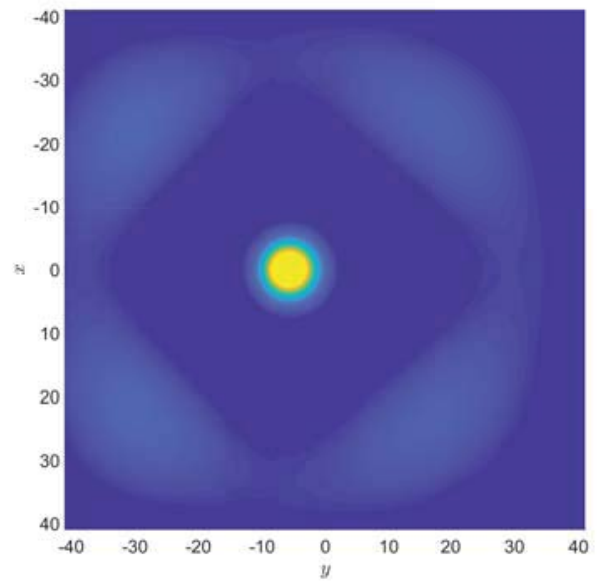

(a)

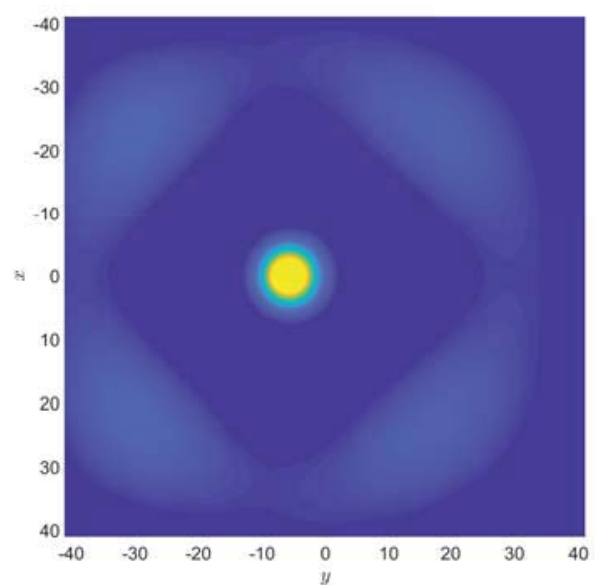

(c)

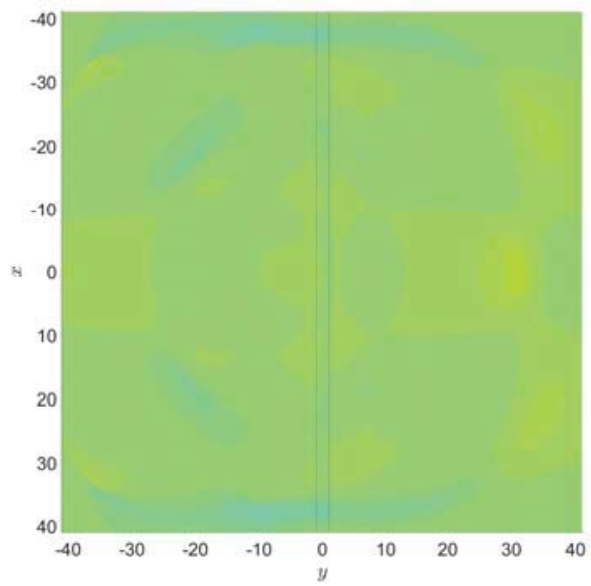

(e)

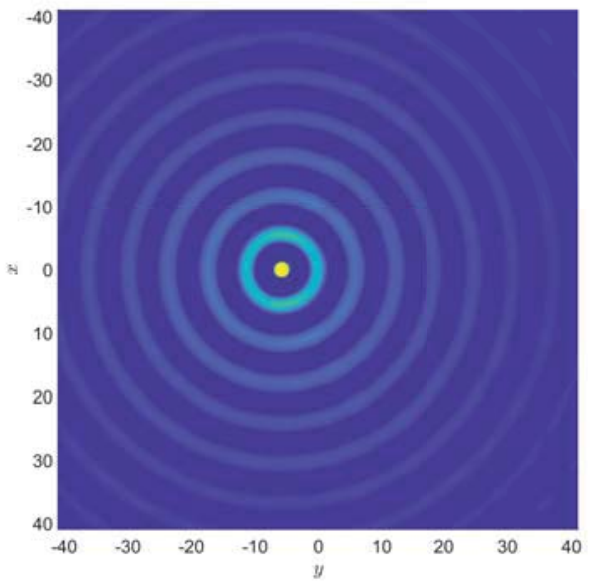

(b)

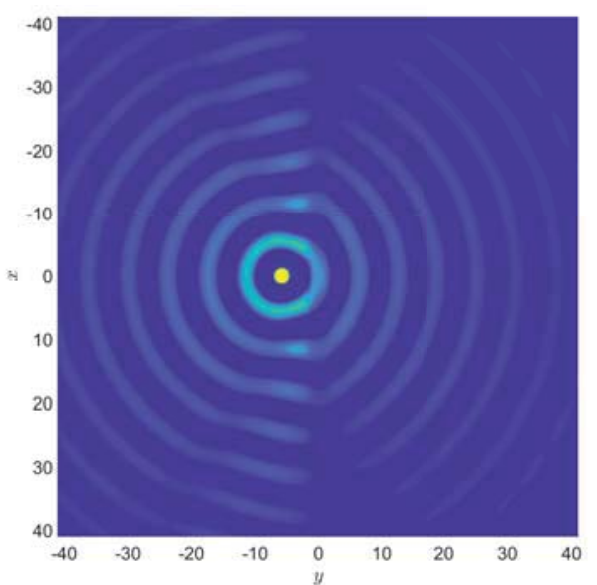

(d)
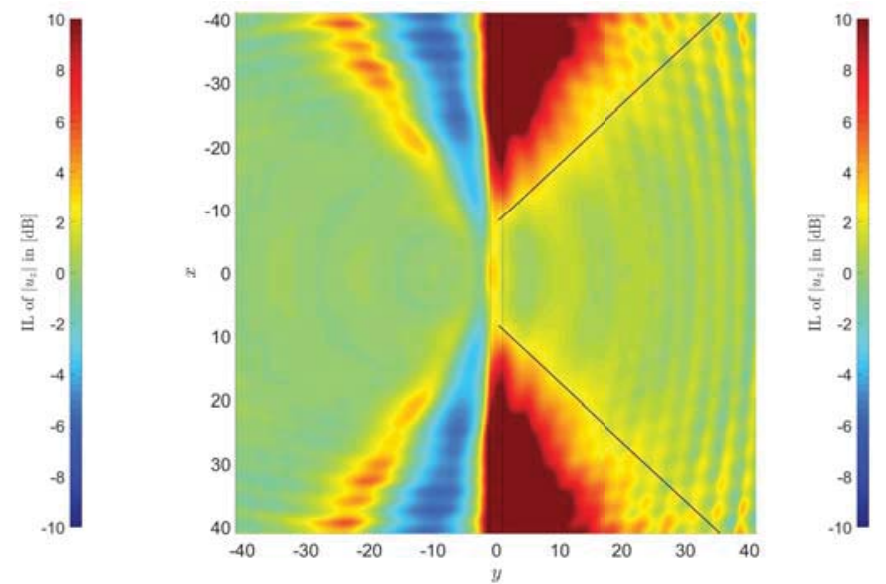

(f)

Figure 6: Real part of the vertical displacement $u_{z}(x, y, z=0, \omega)$ due to harmonic excitation with block load at $y=-6 \mathrm{~m}$ in the reference case $(\mathrm{a}, \mathrm{b})$ and in the case with subgrade stiffening $(\mathrm{c}, \mathrm{d})$ as well as the corresponding insertion loss $\overline{\mathrm{I}}_{\mathrm{z}}(\mathrm{e}, \mathrm{f})$. Left column for $f_{c}>f=5 \mathrm{~Hz}$ and right column for $f_{c}<f=30 \mathrm{~Hz}$. 
the presence of the barrier gives rise to body (P- and $\mathrm{S}-$ ) waves, that are also reflected and transmitted [9]. Furthermore body waves radiating downward the barrier occur, acting like a new wave source at the lower end of the stiff barrier, emitting body waves into the interior of the halfspace [25]. This transformation of Rayleigh waves into body waves is called mode conversion. The screening effect of a stiff barrier with adequate depth therefore is partly based on the reflection of the Rayleigh waves and partly on its transmission into the interior of the halfspace. For fixed dimensions, the decisive parameter for the vibration mitigation efficiency of a stiff barrier is the stiffness difference between soil and infilled trench rather than the impedance [13]. The material damping of the infill material has no significant effect on the on the screening performance [28]. Therefore, very stiff barriers in relatively soft soils have a mitigation efficiency close to that obtained by an open trench [14].

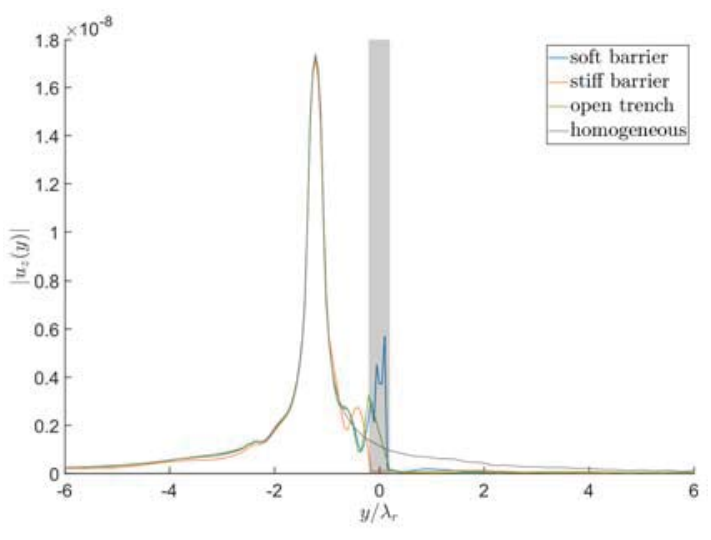

(a)

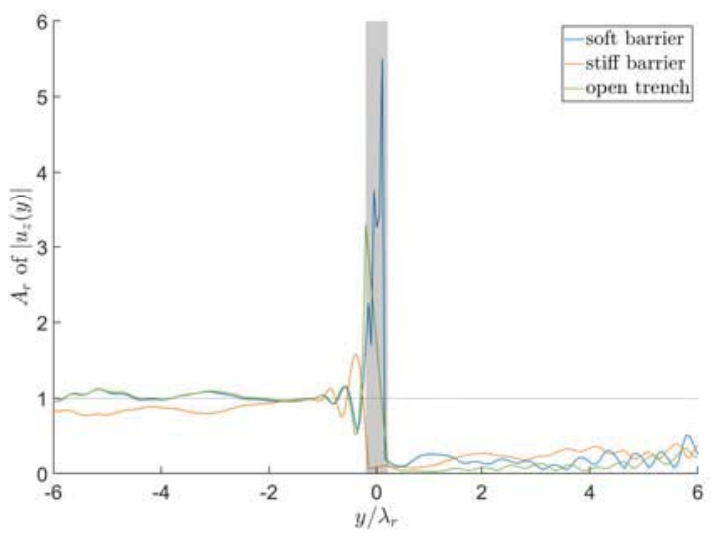

(b)

Figure 7: (a) Absolute value of vertical displacement $\left|u_{z}(x=0, y, z=0, \omega)\right|$ due to harmonic excitation with $f=20 \mathrm{~Hz}$ by a $1 \mathrm{x} 1 \mathrm{~m}$ block load at $y_{\text {load }}=-4 \mathrm{~m}$ for soft barrier, stiff barrier, open trench, homogeneous halfspace and (b) the corresponding amplitude reduction factor $A_{r}$. The gray bar indicates the area enclosed by the open trench resp. the barrier.

Figure 7 a shows the vertical displacement $\left|u_{z}(x=0, y, z=0, \omega)\right|$ for an extremely stiff (Infill C) and a very soft (Infill D) barrier as well as an open trench and the reference case of a homogeneous halfspace (Soil A). The material properties of the barriers and the soil are given in Table 2, the used discretization in Table 1. Again a 1x1 m block load with $P_{0}=1 \frac{\mathrm{N}}{\mathrm{m}^{2}}$ located at $y_{\text {load }}=-4$ was chosen. For the excitation frequency of $20 \mathrm{~Hz}, \lambda_{r}$ results to $3.3 \mathrm{~m}$ and therefore the normalized dimensions of the trench respectively the barrier account for $D_{\mathrm{tr}}=1.2$ and $W_{\mathrm{tr}}=0.4$. The soft barrier and the open trench show large displacement amplitudes at the load sided edge, whereas in case of the stiff barrier the deformation is almost zero. This also reflects in the amplitude reduction factor depicted in Figure 7b, exhibiting $A_{r} \gg 1$ for the open trench and the soft barrier and a very small $A_{r}$ for the stiff barrier. Behind the trench a significant reduction of vibrations is observed for all three cases, albeit the open trench performs best over the largest part of the considered range.

This tendency is also visible in Figures $8(\mathrm{a}, \mathrm{c}, \mathrm{e})$, where the amplitude reduction factor $A_{r}$ over the surface with dimensions normalized by $\lambda_{r}$ is depicted. In the graphs, the area occupied by the barrier is marked by the white lines. The open trench shows the greatest reduction effect over the entire area on the load averted side. Nearly the same pattern of $A_{r}$ develops on the load facing side for the open trench and the soft filled barrier, which can be explained by the similar physical mechanism for the vibration screening, relying almost solely on the reflection of the impinging Rayleigh waves. 


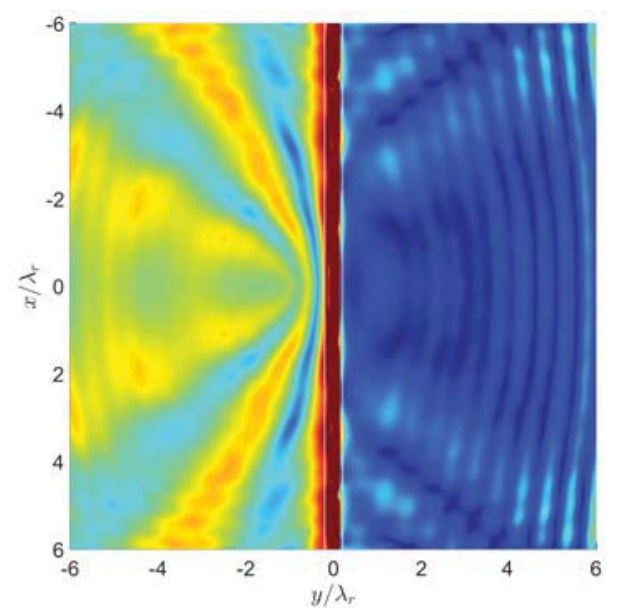

(a)

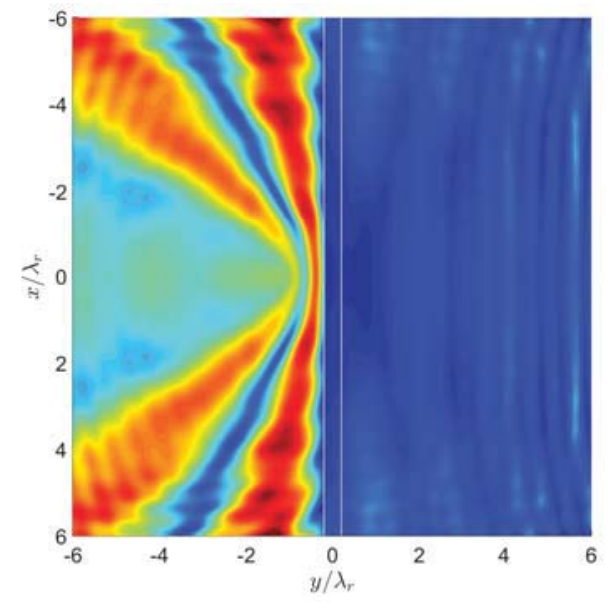

(c)

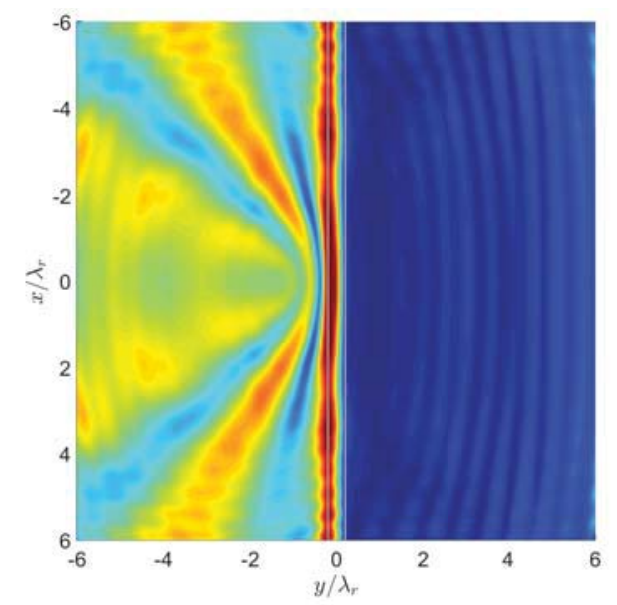

(e)
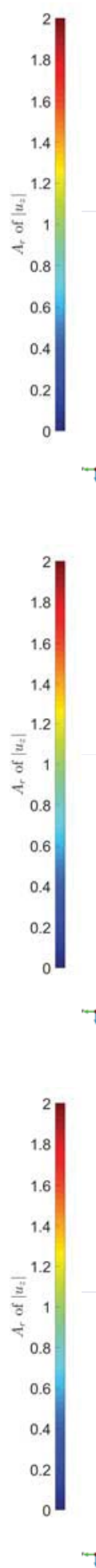

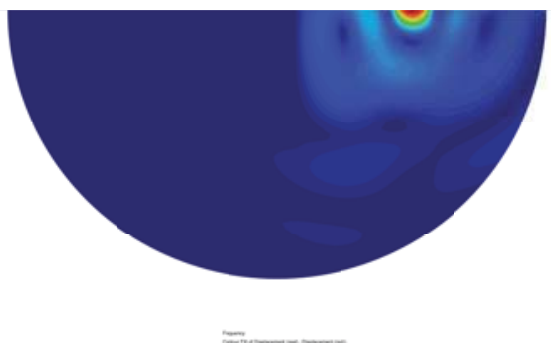

(d)

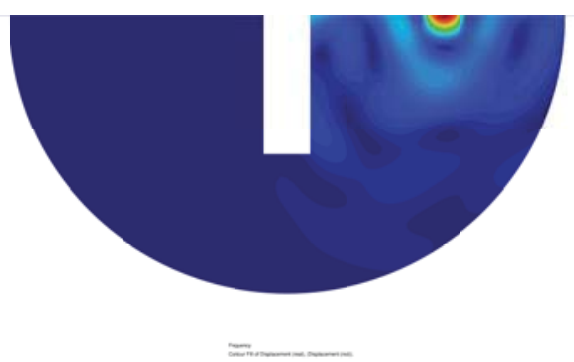

(f)

Figure 8: Left column: Amplitude reduction factor $A_{r}$, right column: Resultant of displacements $\mathbf{u}(x, y, z, \omega)=$ $\sqrt{u_{x}^{2}+u_{y}^{2}+u_{z}^{2}}$ for $(\mathrm{a}, \mathrm{b})$ soft barrier, (c,d) stiff barrier and (e,f) open trench.

However, in case of the stiff barrier, due to the very different ratio of wave velocities in the soil and the barrier compared to the soft infill material as well as larger amount of mode conversion, a very different interference pattern of the reflected waves occurs before the bar- 
rier. Moreover, in Figures $8(\mathrm{~b}, \mathrm{~d}, \mathrm{f})$ the pseudo resultant of the displacements $\mathbf{u}(x, y, z, \omega)=$ $\sqrt{u_{x}^{2}+u_{y}^{2}+u_{z}^{2}}$ within the FEM substructure is illustrated for all three cases in a section at $x=0$. In the considered frequency range the wave lengths in the soil are considerably larger than the barrier width and thus impose their displacements to the barrier quasi statically [14]. It is clearly visible that with the soft filling material, large deformations occur at the edge and within the barrier, similar to the limit case of the reflection of the incident waves at the free end, as for an open trench. In contrast the stiff barrier shows no deformation at all and therefore almost acts as fixed boundary [11].

\subsection{Finite open trench (3D)}

Unlike the previously presented $2.5 \mathrm{D}$ approach, dealing with infinitely long mitigation measures in longitudinal direction, the 3D approach allows the investigation of spatially limited open trenches or barriers, closer to practical applications. For the length invariant structures the energy transmission is restricted over the whole length, whereas in case of finite vibration shielding measures much more complex wave interference phenomena occur, as additionally to the lower trench end diffraction effects arise also at side edges [7]. Thus, regions with reduced or increased vibration amplitudes on the ground surface can clearly be observed as depicted in Figures $10 \mathrm{c}$ and $10 \mathrm{~d}$.

For the investigation of the finite open trench, the three dimensional ITM-FEM approach is applied, whereby the open trench is modeled within the half spherically shaped FEM substructure as displayed in Figure 9. A radius of $R=6 \mathrm{~m}$ was chosen for the sphere and a open trench with $d_{\mathrm{tr}}=3.8 \mathrm{~m}, w_{\mathrm{tr}}=1.2 \mathrm{~m}$ and $l_{\mathrm{tr}}=4.8 \mathrm{~m}$ included. A total region of $B_{x}=B_{y}=64$ m was investigated using $N_{x}=N_{y}=2^{7}$ Fourier series members on the halfspace surface as well as $N_{l}=40$ longitudes and $N_{m}=20$ latitudes for the discretization of the sphere, leading to a finite element size of $d_{x, \mathrm{FE}}=d_{y, \mathrm{FE}}=0.6 \mathrm{~m}$ along the surface. The Material properties of Soil A, given in Table 2, were used for the calculations. In the FEM subsystem solid elements

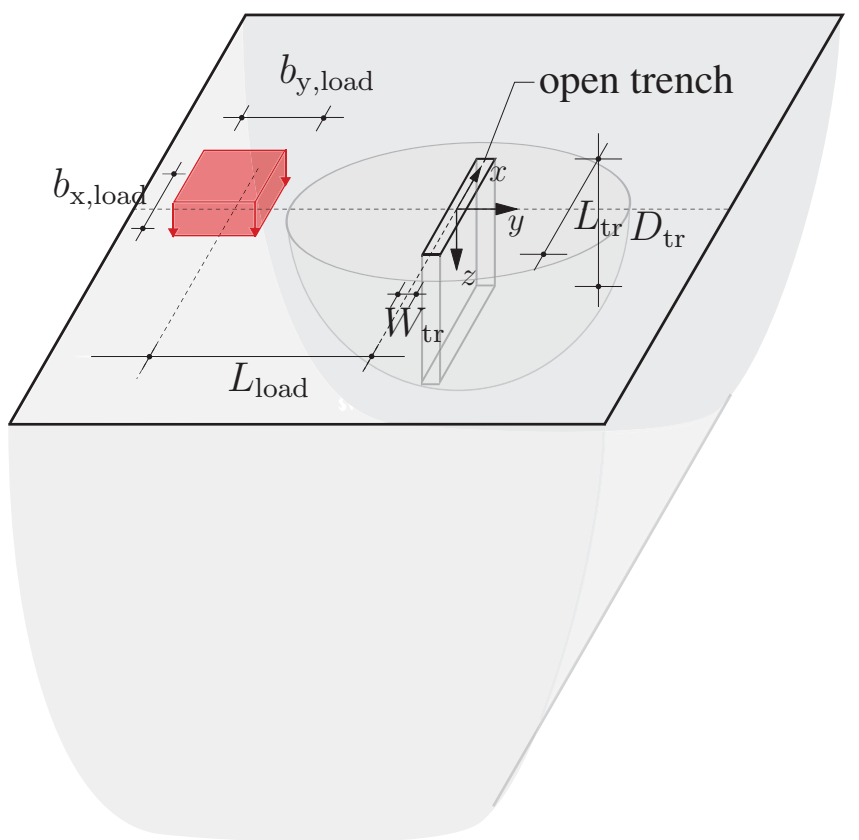

Figure 9: Sketch of the three-dimensional ITM-FEM system for the vibration isolation by an finite open trench. 
with linear shape functions were used, which, in general, are not as accurate as higher order elements, if it comes to stress concentrations or detailed results for the displacement amplitude in the near field around the load are of interest. However, for the assessment of the vibration mitigation efficiency, the response behind the trench is more important than the deformation of the trench itself or the stress concentrations around it [7]. Therefore satisfactory precision is reached for the investigated problem, using the ITM-FEM approach.

In the following sections the influence of the trench length and depth as well as the distance of the load from the trench is investigated and finally compared to results obtained for the infinite trench.

\section{Variation of the excitation frequency}

Figure 10a shows the absolute value of the vertical displacements at $x=0$ over the $y$ due to a harmonic $1.2 \times 1.2 \mathrm{~m}$ block load located at $y_{\text {load }}=-3 \mathrm{~m}$ with excitation frequencies of $12 \mathrm{~Hz}$ and $22 \mathrm{~Hz}$. The corresponding amplitude reduction factor $A_{r}$ is depicted in Figure 10b. As the frequency changes, also the Rayleigh wavelength and therefore the relative dimensions of the open trench vary. At $f=12 \mathrm{~Hz}, \lambda_{r}$ results in $5.5 \mathrm{~m}$ and thus $D_{\mathrm{tr}}=0.69, W_{\mathrm{tr}}=0.22$ and $L_{\mathrm{tr}}=$ 0.87 , whereas for $f=22 \mathrm{~Hz}$ the Rayleigh wave length is $\lambda_{r}=2.9 \mathrm{~m}$ and thereby $D_{\mathrm{tr}}=1.31$, $W_{\mathrm{tr}}=0.41$ and $L_{\mathrm{tr}}=1.65$. The amplitude reduction for $12 \mathrm{~Hz}$, depicted in Figure 10e, is rather limited as a significant part of energy passes below the open trench. With increasing frequency the penetration depth of the Rayleigh wave reduces and therefore the mitigation efficiency rises, as can be seen in Figure 10f showing $A_{r}$ for an excitation frequency of $22 \mathrm{~Hz}$. Amplifications of the displacement amplitudes can be observed in front of the trench and at the sides as well as a reduction behind the trench. For small ratios $l_{\mathrm{tr}} / \lambda_{r}$ amplifications occur also directly behind the open trench, a phenomenon which was also observed by Woods [2] experimentally. Due to the small trench length, especially for lower frequencies, the waves travel around the trench thereby causing this amplifications, as can clearly be seen in Figure 10c. With increasing dimensionless length and depth the mitigation efficiency rises and thus the shadow zone enlarges and gets more defined.

\section{Variation of load position}

Dolling [1] showed that above a certain trench length the shielding effect no longer changes significantly and concluded that an radiation angle of $45^{\circ}-56^{\circ}$ leads to an effective vibration reduction. Also Woods [2] found that larger trenches are required at greater distance from the source to achieve a certain amplitude reduction. Therefore in this section the variation of the load position with a fixed open trench length, depth and width is investigated as depicted in Figure 11a. This leads to a variation of the radiation angle

$$
\alpha=\tan ^{-1}\left(\frac{L_{\mathrm{tr}} / 2}{L_{\mathrm{load}}-W_{\mathrm{tr}} / 2}\right)
$$

of the waves on the open trench of $45^{\circ}$ for $y_{\text {load }}=-3$ to $10^{\circ}$ for $y_{\text {load }}=-14$ and therefore a different shielding zone and mitigation efficiency. Figure $11 \mathrm{~b}$ shows the amplitude reduction factor $A_{r}(x=0, y, z=0, \omega)$ for load positions $y_{\text {load }}=-3,-8,-14$ due to an excitation with $f=22 \mathrm{~Hz}$. With increasing $y_{\text {load }}$ the amplitude reduction at some distance behind the trench decreases substantially, which fits well to Woods's [2] statement, that the screening efficiency is larger, if the trench is positioned close to the source. 


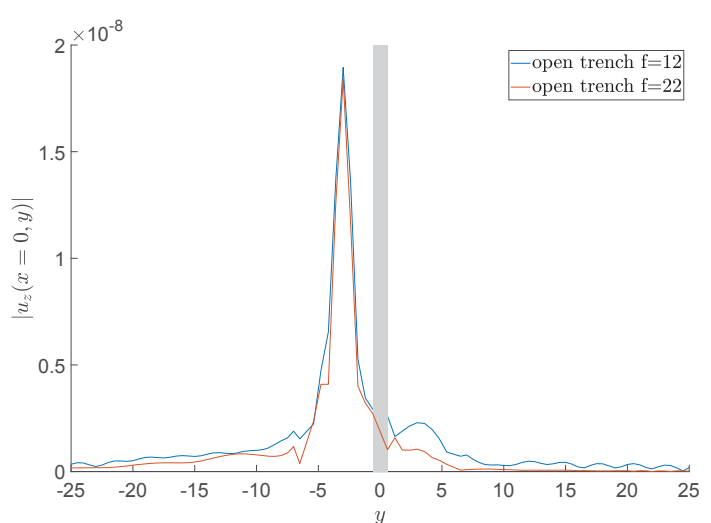

(a)

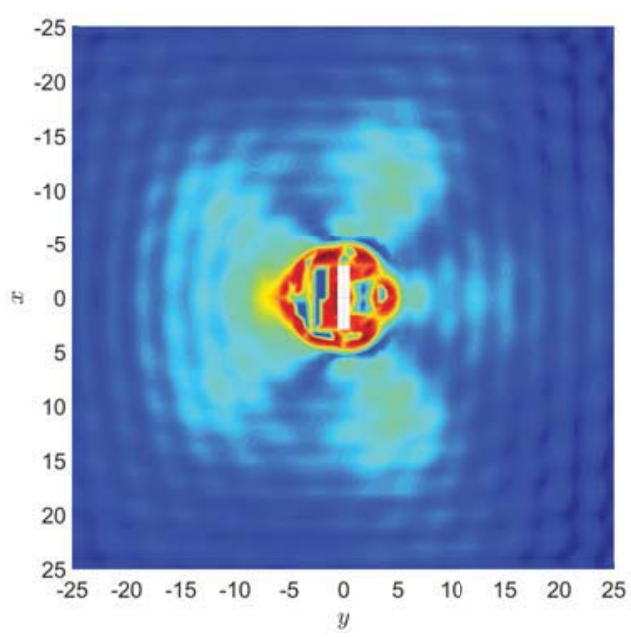

(c)

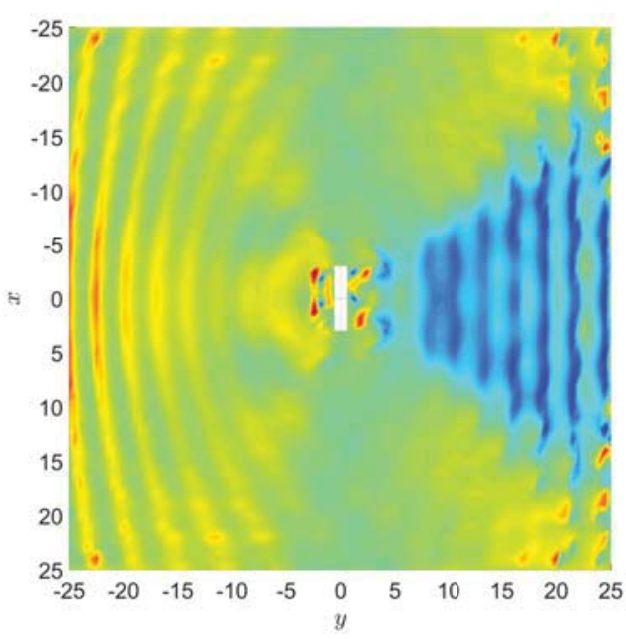

(e)

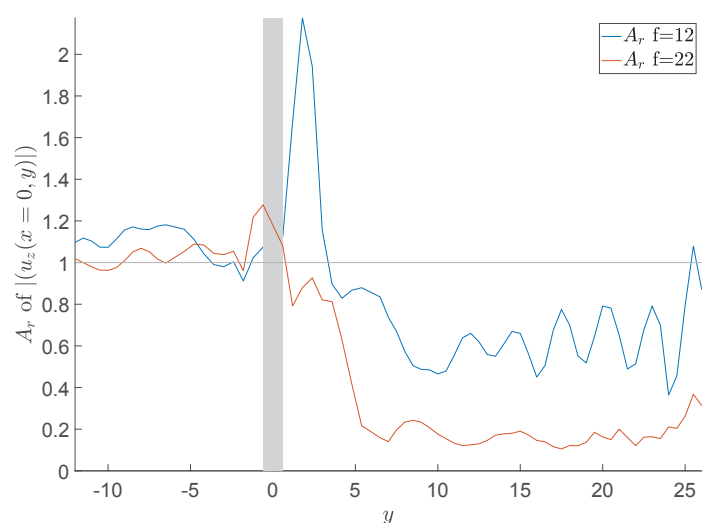

(b)
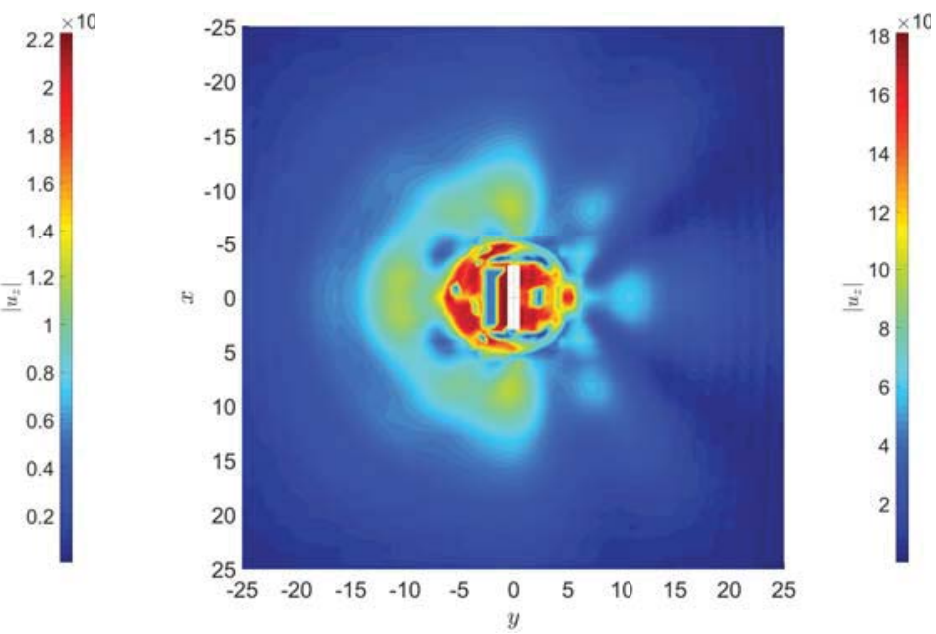

(d)
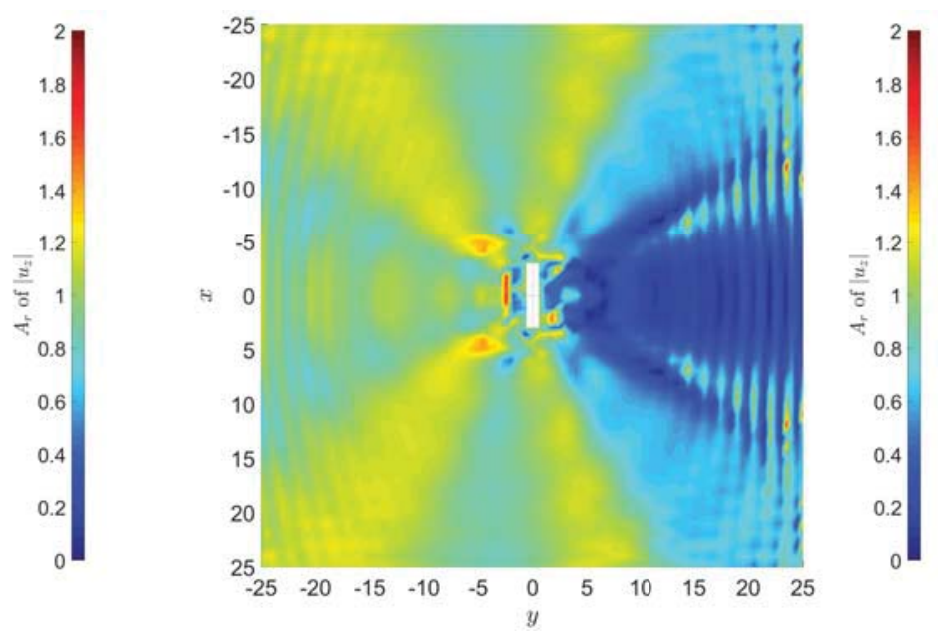

(f)

Figure 10: (a) Absolute value $\left|u_{z}(x=0, y, z=0, \omega)\right|$ and (b) corresponding $A_{r}$. (c,e) Real part of the vertical displacement $u_{z}(x, y, z=0, \omega)$ and $A_{r}$ of $\left|u_{z}(x, y, z=0, \omega)\right|$ for $f=12 \mathrm{~Hz}$ and $(\mathrm{d}, \mathrm{f})$ for $f=22 \mathrm{~Hz}$. 


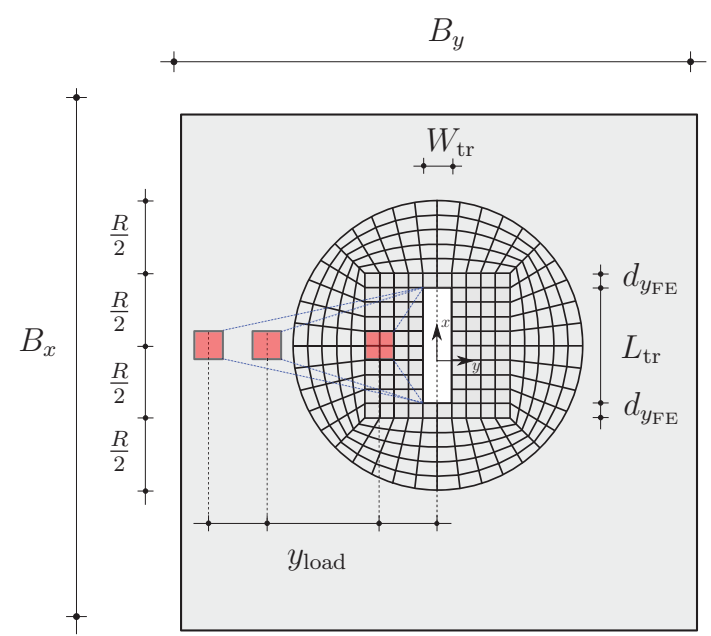

(a)

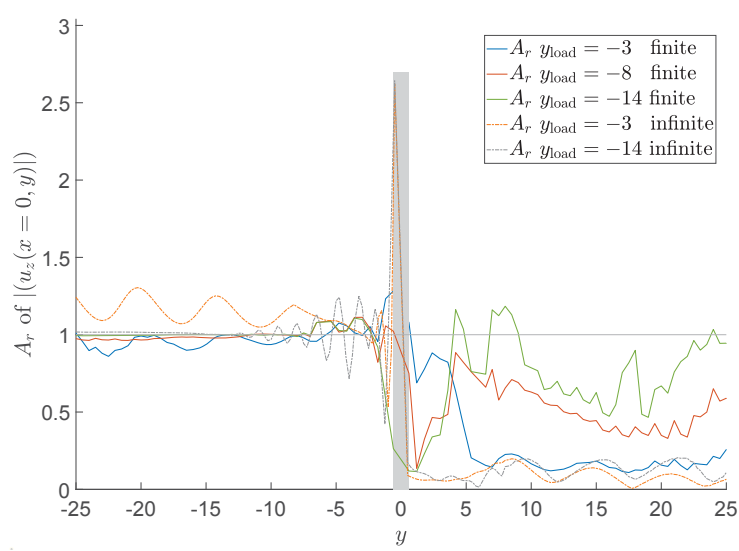

(b)

Figure 11: (a) Setup for variation of load position and radiation angle (blue lines). (b) Amplitude reduction factor $A_{r}$ of $\left|u_{z}(x=0, y, z=0, \omega)\right|$ for different load positions $y_{\text {load }}$ for the finite and the infinite open trench.

This is further confirmed, when the amplitude reduction factors for different load positions considering the infinitely long trench is opposed to the ones obtained for the finite open trench as depicted also in Figure $11 \mathrm{~b}$ as well. The variation of $A_{r}$ due to different load positions is very small for the length invariant open trench because the radiation angle is not relevant in this case, as there is no possibility for the waves to travel around open trench. A more exposed peak in front of the trench can be observed in the $2.5 \mathrm{D}$ case because a bigger part of the incoming waves is reflected leading to a lower $A_{r}$ over the whole length behind the trench.

\section{CONCLUSIONS}

In this paper a coupled Integral Transform Method - Finite Element Method approach for the Soil Structure Interaction of length invariant (2.5D) and finite (3D) structures has been presented. Therefore fundamental solutions of the Lamé differential equation were superposed in order to gain the systems halfspace with partially or fully embedded cylindrical or spherical cavity. At the cavity boundaries a FEM substructure is coupled to the ITM using a direct stiffness coupling by enforcing the continuity of displacements and the equilibrium of forces. The proposed methodology was validated by comparison with the analytical solution of a homogeneous halfspace for the $2.5 \mathrm{D}$ and the $3 \mathrm{D}$ approach.

Within the FEM region, structures with more complex geometry and material distribution can be modeled. This was used to investigate the vibration reduction efficiency of longitudinally infinite open trenches and barriers with soft or stiff infill material. The normalized depth of the barrier and the stiffness contrast between infill material and soil are found to be the most important parameters for the performance of the screening measure. More shallow barriers with less pronounced stiffness contrast, as in case of subgrade stiffening, act as wave impeding barrier up from a critical frequency and provide a significant insertion loss within an area delimited by a critical angle. For deeper stiff or soft filled barriers with distinct stiffness contrast the performance is similar to an open trench.

In case of spatially limited open trenches also the dimensionless length plays an important role. As diffraction occurs at the sides of the trench additionally to the bottom, more complex wave interference patterns occur. The radiation angle and therefore the distance of the source from the trench has fundamental impact on the mitigation efficiency, which is not the case for length invariant trenches. 


\section{REFERENCES}

[1] H.-J. Dolling, Die Abschirmung von Erschütterungen durch Bodenschlitze, Disseration, TU Berlin, Berlin, 1969.

[2] R. D. Woods, Screening of surface waves in soils, Soil mechanics and foundations division (1968) 951-980.

[3] G. Segol, J. F. Abel, P. C. Y. Lee, Amplitude reduction of surface waves by trenches, Journal of the Engineering Mechanics Division 104 (1978) 621-641.

[4] W. A. Haupt, Surface-waves in non-homogeneous halfspace, Proc. Dyn. Meth. in Soil and Rock Mech. (1978) 335-367.

[5] K. Emad, G. D. Manolis, Shallow trenches and propagation of surface waves, Journal of Engineering Mechanics 111 (1985) 279-282.

[6] D. E. Beskos, B. Dasgupta, I. G. Vardoulakis, Vibration isolation using open or filled trenches, Computational Mechanics (1986) 43-63.

[7] B. Dasgupta, D. E. Beskos, I. G. Vardoulakis, Vibration isolation using open or filled trenches part 2: 3-d homogeneous soil, Computational Mechanics 6 (1990) 129-142.

[8] K. L. Leung, D. E. Beskos, I. G. Vardoulakis, Vibration isolation using open or filled trenches, Computational Mechanics 7 (1990) 137-148.

[9] S. Ahmad, T. M. Al-Hussaini, Simplified design for vibration screening by open and in-filled trenches, Journal of Geotechnical Engineering 117 (1991) 67-88.

[10] G. Pflanz, K. Hashimoto, N. Chouw, Reduction of structural vibrations induced by a moving load, Journal of Applied Mechanics 5 (2002) 555-563.

[11] L. Andersen, S. Nielsen, Reduction of ground vibration by means of barriers or soil improvement along a railway track, Soil Dynamics and Earthquake Engineering 25 (2005) 701-716.

[12] M. Adam, O. von Estorff, Reduction of train-induced building vibrations by using open and filled trenches, Computers \& Structures 83 (2005) 11-24.

[13] P. Coulier, S. François, G. Degrande, G. Lombaert, Subgrade stiffening next to the track as a wave impeding barrier for railway induced vibrations, Soil Dynamics and Earthquake Engineering 48 (2013) 119-131.

[14] S. François, M. Schevenels, B. Thyssen, J. Borgions, G. Degrande, Design and efficiency of a composite vibration isolating screen in soil, Soil Dynamics and Earthquake Engineering 39 (2012) $113-127$.

[15] S. François, M. Schevenels, P. Galvín, G. Lombaert, G. Degrande, A 2.5d coupled fe-be methodology for the dynamic interaction between longitudinally invariant structures and a layered halfspace, Computer Methods in Applied Mechanics and Engineering 199 (2010) 1536-1548.

[16] C. F. Long, On the completeness of the lamè potentials, Acta Mechanica 3 (1967) 371-375.

[17] G. Müller, Ein Verfahren zur Erfassung der Fundament-Boden Wechselwirkung unter Einwirkung periodischer Lasten, Dissertation, Technische Universität München, München, 1989.

[18] M. Hackenberg, M. Dengler, G. Müller, Implementation of the finite element method in the fouriertransformed domain and coupling with analytical solutions, in: Cunha A., Caetano E., Ribeiro P., Müller G (Ed.), Eurodyn.

[19] A. C. Eringen, E. S. Suhubi, Elstodynamics, Vol. II.(Linear Theory), Academic Press: New York, 1975.

[20] A. Sommerfeld, Partial differential equations in physics, volume 1 of /Pure and applied mathematics, Acad. Pr, New York, 1964. 
[21] G. Frühe, Überlagerung von Grundlösungen in der Elastodynamik zur Behandlung der dynamischen Tunnel-Boden-Bauwerk-Interaktion, Dissertation, Technische Universität München, München, 2010.

[22] M. Hackenberg, A Coupled Integral Transform Method - Finite Element Method Approach to Model the Soil-Structure-Interaction, Dissertation, Technische Universität München, München, 2016.

[23] J. Freisinger, M. Hackenberg, G. Müller, A coupled integral transform method - finite element method approach to model the soil structure interaction of finite (3d) and length invariant $(2.5 \mathrm{~d})$ systems, Journal of Sound and Vibration 482 (2020) 115443.

[24] J. Freisinger, G. Müller, Modellierung eines halbraums mit sphärischem oder zylinderförmigem hohlraum für dreidimensionale boden-bauwerk-interaktion, Forschung im Ingenieurwesen 83 (2019) 31-42.

[25] W. A. Haupt, Wave propagation in the ground and isolation measures, International Conferences on Recent Advances in Geotechnical Earthquake Engineering and Soil Dynamics (1995).

[26] D. J. Thompson, J. Jiang, M. Toward, M. Hussein, E. Ntotsios, A. Dijckmans, P. Coulier, G. Lombaert, G. Degrande, Reducing railway-induced ground-borne vibration by using open trenches and soft-filled barriers, Soil Dynamics and Earthquake Engineering 88 (2016) 45-59.

[27] P. Coulier, A. Dijckmans, S. François, G. Degrande, G. Lombaert, A spatial windowing technique to account for finite dimensions in $2.5 \mathrm{~d}$ dynamic soil-structure interaction problems, Soil Dynamics and Earthquake Engineering 59 (2014) 51-67.

[28] T. Bose, D. Choudhury, J. Sprengel, M. Ziegler, Efficiency of open and infill trenches in mitigating ground-borne vibrations, Journal of Geotechnical and Geoenvironmental Engineering 144 (2018) 04018048 . 